\title{
Sudden death in epilepsy, surgery, and seizure outcomes: The interface between heart and brain
}

\section{ABSTRACT}

A significant body of literature suggests that sudden unexpected death in epilepsy (SUDEP) has a cardiac mechanism. Although a correlation has been established between freedom from seizures and reduced SUDEP risk, the exact relationship between seizure outcomes following epilepsy surgery and SUDEP is still being studied. Emerging evidence suggests that seizure outcomes following epilepsy surgery and risk for SUDEP may both be governed by a common underlying biologic process linked to cardiac changes and autonomic dysregulation.

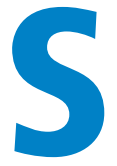

udden unexpected death in epilepsy (SUDEP) is most often defined as the sudden, unexpected, nontraumatic, and nondrowning death of patients with epilepsy. The death may be either witnessed or unwitnessed, and may occur with or without evidence of a seizure, excluding documented status epilepticus. In cases of definite SUDEP, postmortem examination does not reveal a structural or toxicologic cause of death. ${ }^{1}$

The reported incidence of SUDEP ranges from 0.7 to 1.3 cases per 1,000 patient-years in large cohorts of epilepsy patients ${ }^{2,3}$ and from 3.5 to 9.3 cases per 1,000 patient-years in antiepileptic drug registries, medical device registries, and epilepsy surgery programs. ${ }^{4-6}$ SUDEP is currently accepted as the most important epilepsy-related mode of death ${ }^{5,7}$ and is associated with standardized mortality ratios in patients with ongoing seizures as high as 24 to 40 times those of the general population. ${ }^{8}$ The exact mechanism or mechanisms leading to SUDEP remain unknown,,$^{1,5,9,10}$ and despite the identification of several potential risk factors, elimination of seizures still represents the main intervention correlating with risk reduction..$^{5,11}$

This review will first discuss evidence for a cardiac mechanism of SUDEP, then review data traditionally used to support freedom from seizures following epilepsy surgery as a method for reducing SUDEP risk, and

Dr. Jehi reported that she has no financial relationships that pose a potential conflict of interest with this article.

doi:10.3949/ccjm.77.s3.09 finally examine recent evidence suggesting that both SUDEP and seizure outcomes following epilepsy surgery are governed by a common pathway with certain cardiac manifestations.

\section{CENTRAL AUTONOMIC NETWORK}

A tightly interconnected neuronal network controls various elements of the cardiovascular autonomic system. Consideration of a cardiac pathophysiology of SUDEP requires a fundamental understanding of this network (Figure 1). ${ }^{7,12,13}$ The insula, the anterior cingulate gyrus, and the ventromedial prefrontal cortex are key to central cortical control of autonomic function. The insula represents the primary viscerosensory cortex, whereas the cingulate gyrus and prefrontal cortices constitute the premotor autonomic region. At the subcortical level, the hypothalamus provides an interface with endocrine stimuli and triggers autonomic responses to maintain homeostasis. The amygdala, which is an integral component of the limbic system that links the previously mentioned cortical and subcortical centers, mediates the autonomic response to emotions. Beyond their centrality to autonomic control, the insula, amygdala, cingulate gyrus, and prefrontal cortex also represent the most common foci of partial epilepsy, a finding that might explain the frequent observation of autonomic changes in relation to epileptic seizures. ${ }^{14}$

\section{EVIDENCE FOR A CARDIAC MECHANISM OF SUDEP}

The most significant and broadly discussed cardiac mechanism of SUDEP is cardiac arrhythmia brought about by seizure discharges acting through the autonomic nervous system. ${ }^{5,7,15,16}$

\section{Clinical evidence}

A wide spectrum of cardiac arrhythmias-from ictal asystole to atrial fibrillation to repolarization abnormalities to bundle branch blocks- - has been reported during seizures. ${ }^{14-19}$ In one study, ictal cardiac arrhythmias occurred in $42 \%$ of hospitalized epilepsy patients, the most common being an irregular series of abrupt rate changes near the end of the electroencephalographic (EEG) seizure discharge. ${ }^{17}$ In another study, R-R interval analysis dur- 


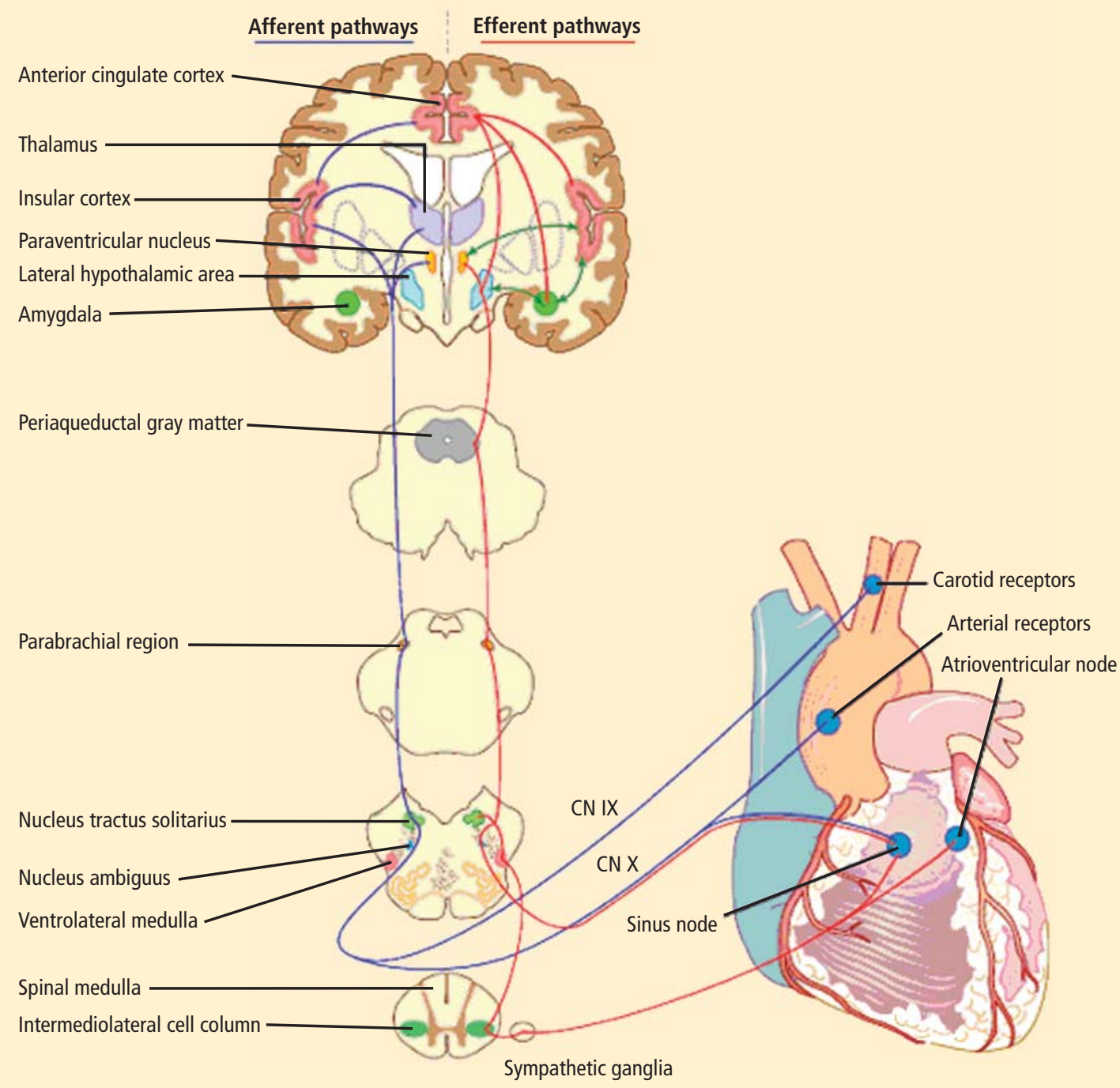

FIGURE 1. The cardiovascular autonomic system. Afferent pathways (violet tracings): The afferent loop of the cardiac autonomic system receives input from chemo- and baroreceptors in the carotid sinus (cranial nerve [CN] IX) and the aortic arch (CN X). Incoming visceral sensations are projected via lamina I of the spinal cord to the nucleus tractus solitarius and, from there, via the parabrachial region to the primary viscerosensitive cortex located in the insula. Efferent pathways (red tracings): The anterior cingulate and orbitofrontal cortex send projections to the hypothalamus and amygdala, but also to the autonomic centers within the brainstem: the periaqueductal gray matter, parabrachial nucleus, nucleus tractus solitarius, nucleus ambiguus, and rostral ventrolateral medulla. Parasympathetic influence on the heart arises primarily from the nucleus ambiguus. Sympathetic output receives tonic excitation from the ventrolateral medulla and projects from the intermediolateral cell columns to the cardiac conduction system and ventricle. Integrated response to emotion and stress (green arrows): The amygdala has reciprocal connections with the cerebral cortex and mediates autonomic response to emotions via projections to the hypothalamus and brainstem. The paraventricular nucleus of the hypothalamus controls internal homeostasis and innervates the autonomic relay centers in the rostral ventrolateral medulla, nucleus tractus solitarius, parabrachial nucleus, and preganglionic vagal and sympathetic neurons. Reprinted from Schuele et al, ${ }^{12}$ which was modified from Britton and Benarroch. ${ }^{13}$

ing the first 10-second period of EEG discharge showed a significant early heart rate increase in $49 \%$ of seizures and an early heart rate reduction in $25.5 \% .^{18}$

Certain clinical seizure characteristics have been correlated with the occurrence of ictal electrocardiographic (ECG) abnormalities. One study found that mean seizure duration was longer in patients with ECG abnormalities than in those without such changes. ${ }^{16}$ Others observed that ictal ECG abnormalities occurred more often and were more severe in generalized tonic-clonic seizures than in complex partial seizures. . $^{15,16,19}$ These same clinical seizure characteristics were correlated with a higher risk of SUDEP, ${ }^{20}$ which suggests an interrelation among seizure semiology, ECG abnormalities, and SUDEP. 
Additionally, direct evidence of seizure-related cardiac changes occurring specifically in SUDEP victims has come from a study that compared EEG and ECG data between 21 SUDEP patients and 43 clinically similar historical controls with refractory partial epilepsy. ${ }^{15}$ The patients who eventually succumbed to SUDEP had a higher ictal maximal heart rate, a greater increase in heart rate with seizures arising from sleep, and a higher incidence of ictal cardiac repolarization and rhythm abnormalities. ${ }^{15}$

\section{Experimental evidence}

Electrical brain stimulation of the limbic system and insular cortex has repeatedly been shown to provoke heart rate changes, including bradycardia, tachycardia, and asystole. ${ }^{14}$ Some studies have even suggested a lateralized influence of the insulae on cardiovascular autonomic control, with intraoperative stimulation of the left posterior insula eliciting a cardioinhibitory response and hypotension, and with stimulation of the right anterior insula eliciting tachycardia and hypertension. ${ }^{7}$ Other studies have suggested that the limbic system has a localization-related influence on cardiovascular responses, with stimulation of the amygdala alone being insufficient to produce the ictal tachycardia so commonly seen in epileptic seizures, which suggests that cortical involvement is essential for the increase in heart rate. ${ }^{21}$ Such cortical stimulation-induced heart rate changes may explain how massive seizure-related discharges can affect cardiac rhythm during the seizure itself.

There is also, however, evidence of a baseline epilepsy-related autonomic dysfunction. Abnormalities in cardiac uptake of meta-iodobenzylguanidine (MIBG) have been demonstrated interictally in patients with chronic temporal lobe epilepsy relative to controls. ${ }^{22} \mathrm{~A}$ recent study specifically showed a pronounced reduction in cardiac MIBG uptake in patients who had ictal asystole compared with other epileptic patients and nonepileptic controls, a finding that suggests a postganglionic cardiac catecholamine disturbance in patients with epilepsy. ${ }^{23}$ The authors proposed that epilepsy-related impairment of sympathetic cardiac innervation limits adjustment and heart rate modulation, and may thus increase the risk of asystole and, ultimately, SUDEP. ${ }^{23}$

\section{EPILEPSY SURGERY AND SUDEP RISK}

The above findings make it reasonable to postulate that recurrent uncontrolled seizures may lead to significant cardiac changes-namely, arrhythmias - which may then lead to a higher risk of SUDEP. Successful epilepsy surgery, the treatment of choice for uncontrolled partial epilepsy, would eliminate seizures and would thus be expected to reduce SUDEP risk.

Several studies support this hypothesis. Reductions in all-cause mortality and in SUDEP following successful epilepsy surgery have been observed when patients who became seizure-free after surgery were compared either with patients who had ongoing postoperative seizures or with patients with intractable epilepsy who did not undergo epilepsy surgery. ${ }^{11,24}$ In one study with a mean follow-up of 3.82 years, no deaths occurred among 199 patients who were seizure-free following epilepsy surgery, whereas there were 11 deaths-including 6 cases of SUDEP — among 194 patients who had persistent seizures following epilepsy surgery. ${ }^{11} \mathrm{~A}$ separate study compared 202 patients who underwent epilepsy surgery with 46 patients with medically treated intractable epilepsy over a mean follow-up period of greater than 5.7 years. ${ }^{24}$ In this study, death occurred in only $7 \%$ of the surgical patients compared with $20 \%$ of the medically treated patients, which suggests that epilepsy surgery (or lack thereof) may be an independent predictor of mortality. Favorable outcome was linked closely to seizure control, as $81 \%$ of the patients who died had 2 or more seizures per year at last follow-up, compared with only $47 \%$ of survivors in the overall cohort. ${ }^{24}$

\section{Complete seizure resolution-not just reduction- seems necessary for SUDEP risk reduction}

In our experience at the Cleveland Clinic Epilepsy Center, we have found that a reduction in seizure frequency following epilepsy surgery is not enough to eliminate SUDEP risk-complete freedom from seizures is required. Of 37 SUDEP cases identified so far in a cohort of 3,481 patients evaluated in our epilepsy monitoring unit between 1990 and 2005 (Jehi et al, in preparation), 7 patients had undergone epilepsy surgery. None of these 7 patients was seizure-free at the time of death; four had a greater than $50 \%$ reduction in seizure frequency. No cases of SUDEP occurred in patients who were seizure-free after surgery.

Our findings mirror earlier results reported by Sperling et al ${ }_{11}^{11}$ who also found that epilepsy surgery improves mortality only when seizures resolve completely, not when they merely "improve." It seems plausible, therefore, that elimination of seizures postoperatively eliminates the risk for the several seizure-related cardiac arrhythmogenic changes discussed above, thereby eliminating the risk for the series of events that eventually leads to SUDEP.

\section{A role for stabilized baseline autonomic control?}

Alternatively, some data suggest that the "autonomic" impacts of epilepsy surgery may extend beyond the immediate seizure-related manifestations to the baseline interictal period in epilepsy patients. One study found that surgery for temporal lobe epilepsy is followed by a reduction of sympathetic cardiovascular modulation and baroreflex sensitivity. ${ }^{25}$ The authors proposed that this finding may be attributable to decreased influences of interictal epileptogenic discharges on brain areas 
involved in cardiovascular autonomic control. These researchers continue to postulate that surgery for temporal lobe epilepsy seems to stabilize cardiovascular control in epilepsy patients by reducing the risk of sympathetically mediated tachyarrhythmias and excessive bradycardiac counterregulation, potentially lowering SUDEP risk. ${ }^{25}$

\section{CARDIAC FINDINGS, SUDEP, AND SEIZURE OUTCOMES}

Whether it is the elimination of seizure-related cardiac arrhythmias achieved by rendering patients seizure-free after surgery, or whether it is the stabilization of baseline autonomic cardiac control by reducing interictal epileptiform discharges, this line of thought assumes that the autonomic dysfunction contributing to SUDEP is caused by epilepsy and that freedom from seizures following epilepsy surgery should therefore be responsible for reducing the risk of death. An alternative hypothesis for the infrequent occurrence of SUDEP in seizure-free patients may be that seizure outcomes following epilepsy surgery and SUDEP risk are actually both governed by the same underlying biologic process. This would suggest that the same patient cohort is at a higher baseline mortality risk and in a prognostically poorer seizure outcome group following epilepsy surgery.

This idea is supported by a recent prospective study among 21 consecutive candidates for temporal lobe epilepsy surgery in which spectral analysis of heart rate variability performed preoperatively was correlated with seizure control 1 year after surgery. ${ }^{26}$ The study found that patients with poor seizure outcomes (Engel class II to IV, signifying ongoing postoperative seizures) had significantly lower power in all domains of heart rate variability than did patients with favorable seizure outcomes. ${ }^{26}$

In another study, heart rate was recorded in 16 patients before and after temporal lobe epilepsy surgery, and sympathetic and parasympathetic cardiac modulation was determined as powers of low-frequency (LF, $0.04-0.15 \mathrm{~Hz}$ ) and high-frequency (HF, 0.15-0.5 Hz) heart rate oscillations. ${ }^{27} \mathrm{The} \mathrm{LF} / \mathrm{HF}$ ratio was calculated as an index of sympathovagal balance. Cardiac MIBG uptake was measured with MIBG single-photon emission computed tomography and compared with control data. Baseline sympathetic LF power and LF/HF ratio were higher in patients who eventually had persistent seizures than in those who became seizure-free. Following surgery, both measures decreased in seizure-free patients but increased in patients with persistent seizures. MIBG uptake was lower in patients than in controls and even lower yet in patients who had persistent seizures. In this subgroup, MIBG uptake declined further after surgery.

Essentially, both of the above studies ${ }^{26,27}$ demonstrate findings of autonomic cardiac abnormalities that predated epilepsy surgery and reliably predicted the eventual surgical outcome in terms of seizure continuance. This suggests that "poor candidates" for epilepsy surgery-ie, those with lower chances of achieving seizure freedom with surgery-may a priori have a higher SUDEP risk. A possible explanation for these findings may be epileptogenic zones, including the insula and other components of the central autonomic network, or molecular/genetic diffuse abnormalities that extend beyond a limited surgically removable seizure focus and involve the heart, increasing the risk for cardiac conduction abnormalities.

\section{CONCLUSIONS}

SUDEP is the most common cause of death in epilepsy patients. A significant body of literature suggests that a cardiac mechanism contributes to its occurrence. Although the exact relationship between seizure outcomes following epilepsy surgery and SUDEP risk is still being investigated, it is accepted that seizure control correlates with reduced mortality. Cardiac changes and autonomic dysregulation seem to be at the "heart" of the problem.

\section{REFERENCES}

1. Nashef L, Hindocha N, Makoff A. Risk factors in sudden death in epilepsy (SUDEP): the quest for mechanisms. Epilepsia 2007; 48:859-871.

2. Nilsson L, Tomson T, Farahmand BY, et al. Cause-specific mortality in epilepsy: a cohort study of more than 9,000 patients once hospitalized for epilepsy. Epilepsia 1997; 38:1062-1068.

3. Tennis P, Cole TB, Annegers JF, et al. Cohort study of incidence of sudden unexplained death in persons with seizure disorder treated with antiepileptic drugs in Saskatchewan, Canada. Epilepsia 1995; 36:29-36.

4. Leestma JE, Annegers JF, Brodie MJ, et al. Sudden unexplained death in epilepsy: observations from a large clinical development program. Epilepsia 1997; 38:47-55.

5. Tomson T, Nashef L, Ryvlin P. Sudden unexpected death in epilepsy: current knowledge and future directions. Lancet Neurol 2008; 7:1021-1031.

6. Nashef L, Fish DR, Sander JW, Shorvon SD. Incidence of sudden unexpected death in an adult outpatient cohort with epilepsy at a tertiary referral centre. J Neurol Neurosurg Psychiatry 1995; 58:462-464.

7. Jehi L, Najm IM. Sudden unexpected death in epilepsy: impact, mechanisms, and prevention. Cleve Clin J Med 2008; 75(suppl 2):S66-S70.

8. Lhatoo SD, Johnson AL, Goodridge DM, et al. Mortality in epilepsy in the first 11 to 14 years after diagnosis: multivariate analysis of a long-term, prospective, population-based cohort. Ann Neurol 2001; 49:336-344.

9. Pedley TA, Hauser WA. Sudden death in epilepsy: a wake-up call for management. Lancet 2002; 359:1790-1791.

10. Schraeder PL, Delin K, McClelland RL, So EL. Coroner and medical examiner documentation of sudden unexplained deaths in epilepsy. Epilepsy Res 2006; 68:137-143.

11. Sperling MR, Feldman H, Kinman J, et al. Seizure control and mortality in epilepsy. Ann Neurol 1999; 46:45-50.

12. Schuele SU, Widdess-Walsh P, Bermeo A, Lüders HO. Sudden unexplained death in epilepsy: the role of the heart. Cleve Clin J Med 2007; 74(suppl 1):S121-S127.

13. Britton JW, Benarroch E. Seizures and syncope: anatomic basis and diagnostic considerations. Clin Auton Res 2006; 16:18-28. 
14. Leung H, Kwan P, Elger CE. Finding the missing link between ictal bradyarrhythmia, ictal asystole, and sudden unexpected death in epilepsy. Epilepsy Behav 2006; 9:19-30.

15. Nei M, Ho RT, Abou-Khalil BW, et al. EEG and ECG in sudden unexplained death in epilepsy. Epilepsia 2004; 45:338-345.

16. Nei M, Ho RT, Sperling MR. EKG abnormalities during partial seizures in refractory epilepsy. Epilepsia 2000; 41:542-548.

17. Blumhardt LD, Smith PE, Owen L. Electrocardiographic accompaniments of temporal lobe epileptic seizures. Lancet 1986; 1:1051-1056.

18. Galimberti CA, Marchioni E, Barzizza F, et al. Partial epileptic seizures of different origin variably affect cardiac rhythm. Epilepsia 1996; 37:742-747.

19. Opherk C, Coromilas J, Hirsch LJ. Heart rate and EKG changes in 102 seizures: analysis of influencing factors. Epilepsy Res 2002; 52:117-127.

20. Langan Y, Nashef L, Sander JW. Case-control study of SUDEP. Neurology 2005; 64:1131-1133.

21. Keilson MJ, Hauser WA, Magrill JP, Goldman M. ECG abnormalities in patients with epilepsy. Neurology 1987; 37:1624-1626.

22. Druschky A, Hilz MJ, Hopp P, et al. Interictal cardiac autonomic dysfunction in temporal lobe epilepsy demonstrated by [ $\left.{ }^{123} \mathrm{I}\right]$ metaiodobenzylguanidine-SPECT. Brain 2001; 124:2372-2382.
23. Kerling F, Dütsch M, Linke R, Kuwert T, Stefan H, Hilz MJ. Relation between ictal asystole and cardiac sympathetic dysfunction shown by MIBG-SPECT. Acta Neurol Scand 2009; 120:123-129.

24. Vickrey BG, Hays RD, Rausch R, et al. Outcomes in 248 patients who had diagnostic evaluations for epilepsy surgery. Lancet 1995; 346:1445-1449.

25. Hilz MJ, Devinsky O, Doyle W, et al. Decrease of sympathetic cardiovascular modulation after temporal lobe epilepsy surgery. Brain 2002; 125:985-995.

26. Persson H, Kumlien E, Ericson M, Tomson T. Preoperative heart rate variability in relation to surgery outcome in refractory epilepsy. Neurology 2005; 65:1021-1025.

27. Hilz MJ, Platsch G, Druschky K, et al. Outcome of epilepsy surgery correlates with sympathetic modulation and neuroimaging of the heart. J Neurol Sci 2003; 216:153-162.

Correspondence: Lara Jehi, MD, Epilepsy Center, Neurological Institute, Cleveland Clinic, 9500 Euclid Avenue, S51, Cleveland, OH 44195; email: jehil@ccf.org 